\title{
Second-Harmonic Generation in Disordered Quadratic Media: Role of a Ferroelectric Domain Structure
}

\author{
V. Roppo ${ }^{1,2}$, K. Kalinowski ${ }^{2}$, W. Wang ${ }^{2}$, C. Cojocaru ${ }^{1}$, J. Trull' ${ }^{1}$, R. Vilaseca ${ }^{1}$, \\ M. Scalora ${ }^{3}$, W. Krolikowski ${ }^{2}$ and Yu. Kivshar ${ }^{2}$ \\ ${ }^{1}$ Departament de Fisica i Enginyeria Nuclear, ETSEIAT, Universitat Politecnica de Catalunya, Colom 11, 08222 Terrassa, Barcelona, Spain \\ ${ }^{2}$ Nonlinear Physics Center and Laser Physics Center, Research School of Physics and Engineering, Australian National University, \\ Canberra ACT 0200, Australia \\ ${ }^{3}$ Charles M. Bowden Research Facility, RDECOM, U.S. Army Aviation and Missile Command, Redstone Arsenal, Alabama 35803, USA \\ email: vito.roppo@upc.edu
}

\begin{abstract}
We study theoretically the second harmonic generation in a nonlinear quadratic crystal with random distribution of ferroelectric domains. We show that the specific features of disordered domain structure greatly affect the emission pattern of the generated harmonics. This phenomenon can be used to characterize the degree of disorder in nonlinear photonic structures.

(C)2010 Optical Society of America

OCIS codes:(190.0190) Nonlinear optics; (190.4420) Nonlinear optics: Nonlinear optics, transverse effects in; (190.2620)

Harmonic generation and mixing
\end{abstract}

Naturally grown ferroelectric crystals such as Strontium Barium Niobate (SBN) often exhibit an irregular multidomain structure with rod-like domains[1]. These disordered nonlinear quadratic crystals have attracted a considerable attention in recent years due to their potential benefits for nonlinear parametric processes [2]. Despite being usually considered as disadvantageous in typical nonlinear optical phenomena due to the low efficiency of the NL process, the randomness of the nonlinear media offers potential applications in broadband frequency generation [3] and ultra-short pulse monitoring [4]. These applications utilize the fact that the crystal with disordered antiparallel domains forms, in fact, a two-dimensional photonic nonlinear structure with an infinite number of $\chi^{2}$ reciprocal vectors corresponding to various local nonlinear gratings. Consequently, such structure enables phase matching of various parametric processes in the same time, over an ultra-broad spectral range and in different spatial directions. Fig.1 represents a schematic illustration of such type of random crystal placed in a typical experimental set-up where the fundamental beam shines the crystal and the SH spatial sidtribution is recorded. The inverted ferroelectric domains are represented by bright and dark colors, respectively. However, recent experiments with random domain SBN crystals also demonstrated that the emission pattern of the generated waves may differ drastically from sample to sample, being either spatially homogeneous or exhibiting distinct intensity peaks $[3,6]$. This behavior points towards the difference in the actual distributions of ferroelectric domains in different samples. In fact, observations of random domains ranging from tens of nanometers to few microns have been reported in the literature [1].

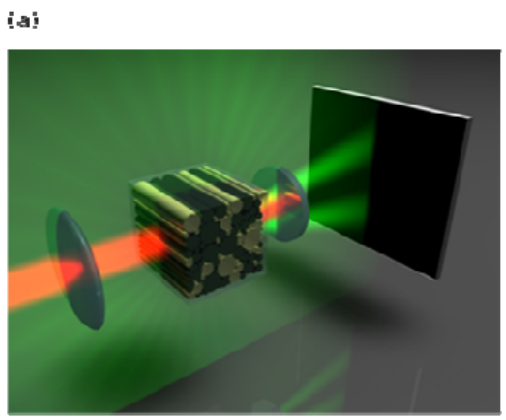

ib:

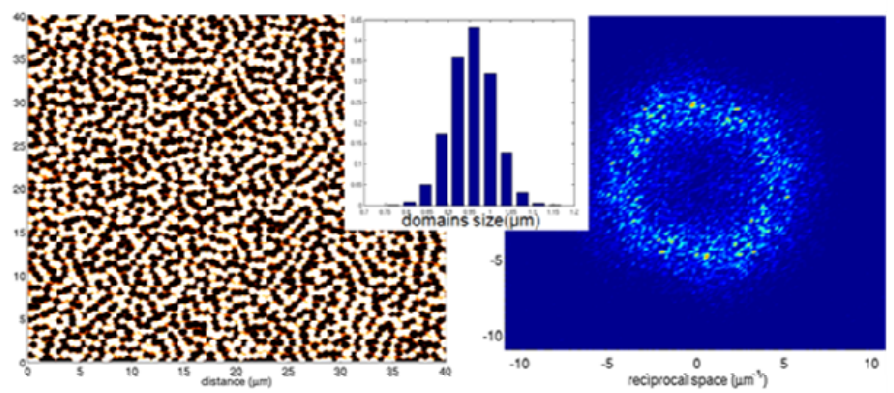

Fig.1. (a) Schematic representation of the second harmonic generation in multi-domain disordered structure in ferroelectric crystal. (b) Example of two-dimensional domain distribution and (c) its spatial Fourier spectrum. Inset: domain size distribution with the mean value of a $=950 \mathrm{~nm}$

In order to obtain a better understanding of the nonlinear interaction in random media, in this work we analyze theoretically and numerically the role of the domain distribution on the efficiency and transverse characteristics of the SH emission in a quadratic random crystal. To our knowledge this is the first attempt to 
approach this problem in a two dimensions environment. This is a crucial point due to the combination of diffraction and scattering processes that take place in the interaction.

The first step is the modeling of the random domain pattern. Different structures are generated and tested with different statistical parameters (see for example Fig.2). Due to their two-dimensional nature, this will allow to diagram the proper $2 \mathrm{D}$ map of the reciprocal vectors possessed by in the structure. At this stage, it is possible to forecast different SH scenarios with the classical phase matching schemes. In the second step a theoretical study based on statistical argumentation will lead to an estimation of the type of SH emission using parameters as main domain size $\left(\rho_{0}\right)$ and distribution variance $(\sigma)$. The analytical treatment is a 2D extension of the work on Ref.[7].

Finally, we numerically solve the full vectorial 2D Maxwell's equations including the actual domain patterns as quadratic nonlinear source in the sample and allowing for fast envelope modulation, scattering and diffraction. Furthermore the role of the light scattering due to the index jump at the domains' walls is included and discussed. In Fig.3 is reported the output of one of these simulations. The SH emissions obtained in the numerical simulations are supported by our experimental evidences[5]. All the results obtained are in very good agreement with the statistical analytical study demonstrating that there is direct connection between the statistical properties of the domain structure and the far field of the second harmonic. This effect can be employed to characterize the degree of randomness of the quasi-phase matching domain pattern.

$|\mathrm{H}|^{2}$ fields (a.u.)
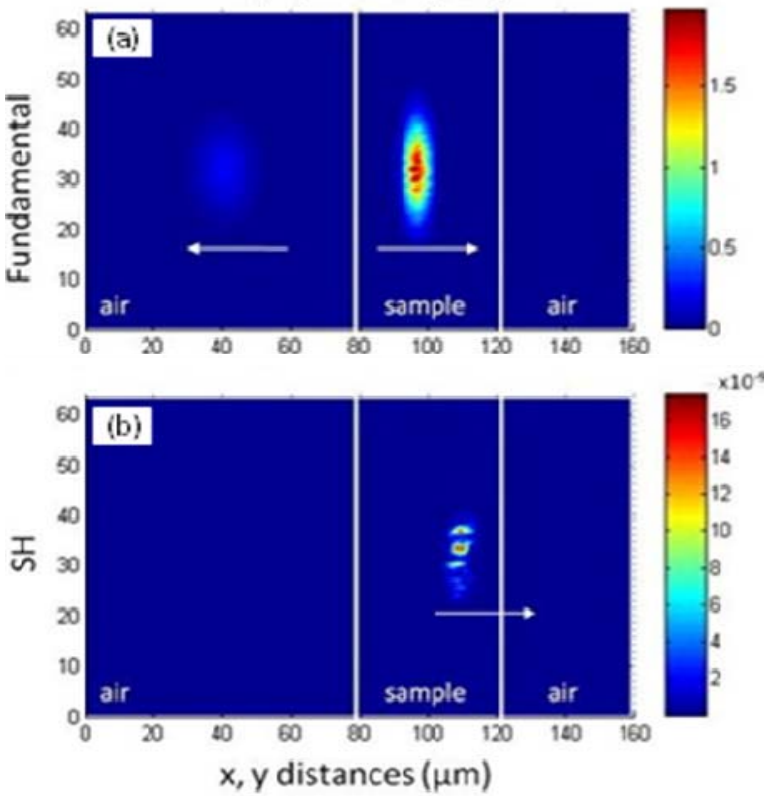

K-spectra (a.u.)
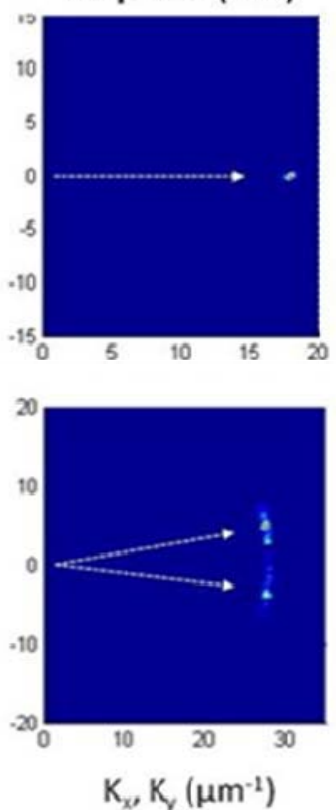

Energies(a.u.)
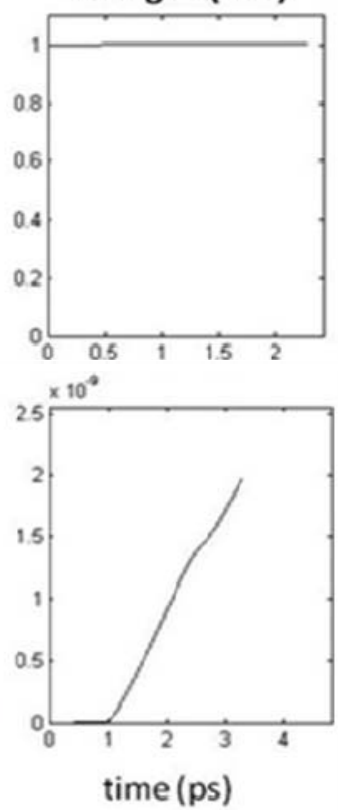

Fig.3: Numerical simulations - generation of the second harmonic by a femtosecond laser pulse in a disordered structure. A fundamental pulse in initially placed in the air and propagates toward the sample in the right direction. When it reaches the first air/sample interface a portion is backward reflected and the remaining part keep propagating forward. Due to the presence of the nonlinearity a SH pulse is generated and propagates forward. Left column: Spatial intensity distribution of the fundamental (a) and second harmonics (b) in the case $\rho_{0}=0.3 \mu \mathrm{m}, \sigma=0.05 \mu \mathrm{m}$, $\lambda_{\mathrm{F}}=0.800 \mu \mathrm{m}$. Middle column - the structure of the interacting waves in the spatial Fourier space. Right column - energy of the fundamental and second harmonics as a function of the time of propagation.

\section{References}

[1] R. V. Gainutdinov, T. R. Volk, O. A. Lysova, I. I. Razgonov, A. L. Tolstikhina and L. I. Ivleva, "Recording of domains and regular domain patterns in strontium barium niobate crystals in the field of atomic force microscope," Appl. Phys. B 95, 505-512 (2009).

[2] M. Baudrier-Raybaut, R. Haidar, Ph. Kupecek, Ph. Lemasson, and E. Rosencher, "Random quasi-phase-matching in bulk polycrystalline isotropic nonlinear materials", Nature 432, 374-376 (2004).

[3] R. Fischer, D. N. Neshev, S. M. Saltiel, W. Krolikowski and Yu. S. Kivshar, "Broadband femtosecond frequency doubling in random media", Appl. Phys. Lett. 89, 191105-1-191105-3 (2006).

[4] R. Fischer, D. N. Neshev, S. M. Saltiel, A. A. Sukhorukov, W. Krolikowski and Yu. S. Kivshar, "Monitoring ultrashort pulses by the transverse frequency doubling of counterpropagating pulses in random media", Appl. Phys. Lett. 91, 031104-1-031104-3 (2007).

[5] V. Roppo, D. Dumay, J. Trull, C. Cojocaru, S. M. Saltiel, K. Staliunas, R. Vilaseca, D. N. Neshev,W. Krolikowski and Yu. S. Kivshar, "Planar second-harmonic generation with noncollinear pumps in disordered media," Opt. Express 16, 14192-14199 (2008).

[6] J. Trull, C. Cojocaru, R. Fischer, S. M. Saltiel, K. Staliunas, R. Herrero, R. Vilaseca, D. N. Neshev, W. Krolikowski and Yu. S. Kivshar, "Second-harmonic parametric scattering in ferroelectric crystals with disordered nonlinear domain structures," Opt. Express 15, 15868-15877 (2007).

[7] Y. Le Grand, D. Rouede, C. Odiu, R. Aubry, and S. Mattauch, "Second-harmonic scattering by domains in RbH2PO4 ferroelectric," Opt. Commun. 200, 249-260 (2001). 